DOI: https://doi.org/10.34069/AI/2021.43.07.13

How to Cite:

Gerasimova , I., Nesterenko, N., \& Tkachenko, I.. (2021). Statistical assessment of the health of the Russian population. Amazonia Investiga, 10(43), 133-140. https://doi.org/10.34069/AI/2021.43.07.13

\title{
Statistical assessment of the Russian population's health
}

\section{Статистическая оценка здоровья населения России}

Received: May 28, 2021

Accepted: July 17, 2021

\begin{abstract}
The article presents the results of modeling selfassessment of individuals' health depending on a number of economic and social factors. The information base of the research is the data of the Comprehensive Survey of Living Conditions of the Population in the Russian Federation for 2018 by Rosstat. A multinomial regression model was used as a toolkit. It was found that subjective assessment of health is significantly influenced by gender, age, marriage status, employment, work in an area of specialization similar to that obtained, nervous tension, harmful factors, and other inconveniences in the workplace, hazardous nature of work, dissatisfaction with wages and job reliability, inability to live an active life, health problems, smoking and alcohol consumption. The results can be used both to adjust social policies and by individuals themselves to improve their health assessments.
\end{abstract}

Keywords: subjective health, self-rated health, multinomial regression.

\begin{abstract}
Аннотация
В статье приводятся результаты моделирования самооценки здоровья индивидов в зависимости от ряда экономических и социальных факторов. Информационной базой исследования являются данные Комплексного наблюдения Росстата условий жизни населения за 2018 год в Российской Федерации. В качестве инструментария использована модель мультиномиальной регрессии. Выявлено, что значимое влияние на субъективную оценку здоровья оказывают пол, возраст, состояние в зарегистрированном браке, работа по найму, работа по специальности, близкой к полученной, нервное напряжение, вредные факторы и другие неудобства на рабочем месте, опасный характер работы, неудовлетворенность заработной платой и надежностью работы, невозможность вести активную жизнь, наличие проблем со здоровьем, курение и употребление алкоголя. Полученные результаты могут быть использованы как для корректировки социальной политики, так и самими индивидами для улучшения оценок собственного здоровья.
\end{abstract}

Ключевые слова: субъективное здоровье, самооценка здоровья, мультиномиальная регрессия.

\footnotetext{
${ }^{49} \mathrm{PhD}$ in Economics, Associate Professor, Rostov State University of Economics, Russia.

${ }^{50}$ Associate Professor, Rostov State Economic University, Russia.

${ }^{51} \mathrm{PhD}$ in Economics, Associate Professor, Rostov State Economic University, Russia.
} 


\section{Introduction}

Population health is one of the most important economic resources (Fafard and Cassola, 2020; Nusratullin et al, 2020). In addition to objective characteristics of population health, how individuals assess their health is also important. Although there is a correlation between objective and subjectively perceived health, there are also differences in their assessments. At the same time, it is the self-assessment of health that largely determines the behavior of an individual, his economic and social activity (Rodrigues et al, 2021). Therefore, the study of the determinants of subjectively perceived health of the population is an urgent task.

The purpose of this research is to build a model of self-assessment of the health of individuals in Russia, depending on a number of economic and social factors, and to interpret its results. The following tasks are set to achieve the goal:

1) Conduct a theoretical review of the literature regarding the construction of models of selfassessment of citizens' health;

2) Construct a model of self-assessment of individual health in Russia depending on a number of economic and social factors;

3) Interpret the results of the constructed model of self-assessment of the health of individuals in Russia.

The information base of the research is the data of the Comprehensive Survey of Living Conditions of the Population in the Russian Federation for 2018 by Rosstat (Federal State Statistics Service of the Russian Federation, 2019). A multinomial regression model was used as an instrument.

\section{Literature Review}

In modern socio-economic conditions, the quality of life is one of the main criteria for assessing the state of health of the population. The World Health Organization today focuses on improving the quality of life for all categories of the population (Andreev et al, 2003 Shevchuk et al, 2020). This is due to the increased interest in the quality of life in recent years, associated with the growth of crises in many countries around the world, which is reflected in the health of the population (Verropoulou, 2012).

Currently, in addition to traditional indicators, there are social characteristics of health, including: the subjective attitude of individuals to their health, social attitudes and self- preservation behavior of people. Health SelfAssessment (HSA) is an integral indicator that reflects a person's subjective feelings about his or her health and is widely used in health research (Perlman and Bobak, 2008). The ease of using one or more questions to obtain sufficiently reliable information has attracted the attention of researchers who are trying to find factors suitable for assessing population health in mass surveys.

The diagnostic and prognostic significance of HSA has been discussed in the literature for more than five decades, mainly because this indicator is associated with various health indicators and life expectancy. There are many works that reflect efforts to objectify it (Borim et al, 2017). Thus, in the work of Puvil et al. (2016) HSA scores were compared to physical and mental health in a population of 85-year-olds living in the Netherlands. Various associations of selfreported health, including mortality, were assessed. Earlier evidence has shown that HSA results in most cases coincide with objective measures of health, such as medical examinations (French et al, 2012).

Under these circumstances, the use of the HSA to determine the prognosis of life and health in this population is very attractive, as evidenced by the sufficient number of publications. At the same time, research shows that people assess their health differently, realizing that illnesses and disabilities are more common at older ages. Indeed, as researchers have shown, the relationship between physical health and HSA becomes less pronounced with age (Heller et al, 2009). HSA partly reflects a condition that does not account for objective measures of health. The burden of diseases of the older generation falls mostly on the shoulders of the surrounding people, the health care system and social welfare institutions. Therefore, HSA issues in the elderly have a pronounced practical relevance (Borim et al, 2014).

Large studies around the world have also shown that HSA is associated with mortality and morbidity (Nery Guimarães et al, 2012).

\section{Methodology}

To model the subjective assessment of health, we used data from the 2018 Rosstat Comprehensive Living Conditions Survey of the population, obtained from the questionnaire for individuals (Federal State Statistics Service of the Russian Federation, 2019). 


\section{AMAZONDA \\ 1กvestiga}

The general characteristics of the respondents are as follows:

1) $59.6 \%$ of respondents are in a registered marriage, $12.7 \%$ have never been married, $13.8 \%$ are divorced, and $7.2 \%$ are in an unregistered marriage;

2) the vast majority (79.2\%) work in an enterprise or organization with the status of a legal entity. $12.5 \%$ work at enterprises of individual entrepreneurs, $2.4 \%$ are entrepreneurs, $2.9 \%$ work on an individual basis, $1.9 \%$ are employed by private persons, $0.6 \%$ work on the farm and $0.4 \%$ work in their own household;

3) Most of the respondents (46\%) assessed their health as satisfactory, $47.3 \%$ assessed their health as good. Very good health is assessed by $4.4 \%$ of the respondents, $2.2 \%$ assessed their health as bad and only $0.1 \%$ assessed their health as very bad. Thus, in general, the health of the respondents is assessed at an average level.

Since the dependent variable (self-assessment of health) is ordinal and takes five variants of values, from "very poor" to "very good," an ordinal regression model was suitable for modeling. However, according to the test for parallelism of lines (chi-square equal to 815.185), the hypothesis of parallelism is rejected at the significance level of 0.001 , which does not allow using an ordinal regression model to assess risks of negative self-assessment of health.

Therefore, a multinomial regression model was estimated to assess the risk of negative selfreported health, as presented in Table 1 (Appendix A). The model is statistically significant, the values of pseudo-R-square from 0.3 to 0.4 testify that the model explains the variation of health self-assessment depending on factors well enough.

The dependent variable was the result of respondents' evaluation of their health, with the options of "very good", "good", "satisfactory", "poor", and "very poor". The category "very good" serves as the benchmark.

Table 1.

Results of multinomial regression model evaluation with the dependent variable "subjective health assessment"

\begin{tabular}{|c|c|c|c|c|c|c|c|c|}
\hline \multirow{2}{*}{ Factors } & \multicolumn{2}{|l|}{ Good } & \multicolumn{2}{|l|}{ Satisfactory } & \multicolumn{2}{|l|}{ Poor } & \multicolumn{2}{|l|}{ Very poor } \\
\hline & Coefficient & $\operatorname{Exp}(B)$ & Coefficient & $\operatorname{Exp}(B)$ & Coefficient & $\operatorname{Exp}(B)$ & Coefficient & $\operatorname{Exp}(B)$ \\
\hline 1 & 2 & 3 & 4 & 5 & 6 & 7 & 8 & 9 \\
\hline Free member & $\begin{array}{l}0,389 \\
(1,223)\end{array}$ & - & $\begin{array}{l}-0,599 \\
(1,221)\end{array}$ & - & $\begin{array}{l}-4,341 * * * \\
(1,565)\end{array}$ & - & $\begin{array}{l}-34,139 \\
(9212,183)\end{array}$ & - \\
\hline Age & $\begin{array}{l}0,043 * * * \\
(0,003)\end{array}$ & 1,044 & $\begin{array}{l}0,107 * * * \\
(0,003)\end{array}$ & 1,113 & $\begin{array}{l}0,12 * * * \\
(0,005)\end{array}$ & 1,127 & $\begin{array}{l}0,082 * * * \\
(0,019)\end{array}$ & 1,085 \\
\hline $\begin{array}{l}\text { Gender } \\
\text { (female) }\end{array}$ & $\begin{array}{l}-0,5 * * * \\
(0,054)\end{array}$ & 0,607 & $\begin{array}{l}-0,748 * * * \\
(0,057)\end{array}$ & 0,474 & $\begin{array}{l}-0,916 * * * \\
(0,105)\end{array}$ & 0,4 & $\begin{array}{l}-0,78^{*} \\
(0,473)\end{array}$ & 0,458 \\
\hline \multicolumn{9}{|c|}{ Marital status (never married) } \\
\hline $\begin{array}{l}\text { Is in a } \\
\text { registered } \\
\text { marriage }\end{array}$ & $\begin{array}{l}0,323 * * * \\
(0,058)\end{array}$ & 1,381 & $\begin{array}{l}0,351 * * * \\
(0,064)\end{array}$ & 1,421 & $\begin{array}{l}0,233 \\
(0,149)\end{array}$ & 1,262 & $\begin{array}{l}0,289 \\
(0,835)\end{array}$ & 1,335 \\
\hline $\begin{array}{l}\text { Is in an } \\
\text { unregistered } \\
\text { marriage }\end{array}$ & $\begin{array}{l}0,116 \\
(0,087)\end{array}$ & 1,123 & $\begin{array}{l}0,122 \\
(0,095)\end{array}$ & 1,129 & $\begin{array}{l}0,355^{*} \\
(0,191\end{array}$ & 1,427 & $\begin{array}{l}1,41 \\
(0,894)\end{array}$ & 4,096 \\
\hline $\begin{array}{l}\text { Widower/Wid } \\
\text { ow }\end{array}$ & $\begin{array}{l}-0,095 \\
(0,176)\end{array}$ & 0,91 & $\begin{array}{l}-0,16 \\
(0,18)\end{array}$ & 0,852 & $\begin{array}{l}-0,521 * * \\
(0,246)\end{array}$ & 0,594 & $\begin{array}{l}1,173 \\
(0,956)\end{array}$ & 3,233 \\
\hline Divorced & $\begin{array}{l}0,033 \\
(0,083)\end{array}$ & 1,033 & $\begin{array}{l}-0,022 \\
(0,089)\end{array}$ & 0,978 & $\begin{array}{l}-0,093 \\
(0,175)\end{array}$ & 0,911 & $\begin{array}{l}0,305 \\
(0,912)\end{array}$ & 1,356 \\
\hline Separated & $\begin{array}{l}-0,248 \\
(0,152)\end{array}$ & 0,781 & $\begin{array}{l}-0,292 * \\
(0,163)\end{array}$ & 0,747 & $\begin{array}{l}-0,084 \\
(0,296)\end{array}$ & 0,919 & $\begin{array}{l}0,928 \\
(1,136)\end{array}$ & 2,53 \\
\hline $\begin{array}{l}\text { Main job (In the } \\
\text { In an } \\
\text { enterprise, in } \\
\text { an }\end{array}$ & r own househ & & & & & & & \\
\hline $\begin{array}{l}\text { an } \\
\text { organization } \\
\text { with the status } \\
\text { of a legal } \\
\text { entity }\end{array}$ & $\begin{array}{l}-0,71 \\
(0,469)\end{array}$ & 0,492 & $\begin{array}{l}-0,36 \\
(0,484)\end{array}$ & 0,698 & $\begin{array}{l}-0,32 \\
(0,673)\end{array}$ & 0,726 & $\begin{array}{l}15,197 \\
(5306,674)\end{array}$ & 3980247 \\
\hline $\begin{array}{l}\text { At the } \\
\text { enterprise of } \\
\text { an individual } \\
\text { entrepreneur }\end{array}$ & $\begin{array}{l}-0,575 \\
(0,472)\end{array}$ & 0,563 & $\begin{array}{l}-0,173 \\
(0,487)\end{array}$ & 0,841 & $\begin{array}{l}0,03 \\
(0,68)\end{array}$ & 1,03 & $\begin{array}{l}15,212 \\
(5306,74)\end{array}$ & 4041084 \\
\hline
\end{tabular}




\begin{tabular}{|c|c|c|c|c|c|c|c|c|}
\hline On the farm & $\begin{array}{l}-0,614 \\
(0,557)\end{array}$ & 0,541 & $\begin{array}{l}-0,277 \\
(0,577)\end{array}$ & 0,758 & $\begin{array}{l}0,243 \\
(0,8)\end{array}$ & 1,275 & $\begin{array}{l}0,052 \\
(6312,3)\end{array}$ & 1,053 \\
\hline 1 & 2 & 3 & 4 & 5 & 6 & 7 & 8 & 9 \\
\hline $\begin{array}{l}\text { In the field of } \\
\text { entrepreneuria } \\
1 \text { activity }\end{array}$ & $\begin{array}{l}-0,949 * * \\
(0,481)\end{array}$ & 0,387 & $\begin{array}{l}-0,783 \\
(0,499)\end{array}$ & 0,457 & $\begin{array}{l}-0,877 \\
(0,725)\end{array}$ & 0,416 & $\begin{array}{l}14,55 \\
(5306,7)\end{array}$ & 2084457 \\
\hline $\begin{array}{l}\text { Employed by } \\
\text { private } \\
\text { households } \\
\text { (individuals) }\end{array}$ & $\begin{array}{l}-1,09 * * \\
(0,025)\end{array}$ & 0,336 & $\begin{array}{l}-0,567 \\
(0,504)\end{array}$ & 0,567 & $\begin{array}{l}-0,353 \\
(0,713)\end{array}$ & 0,702 & $\begin{array}{l}14,548 \\
(5306,7)\end{array}$ & 2079938 \\
\hline $\begin{array}{l}\text { On an } \\
\text { individual } \\
\text { basis }\end{array}$ & $\begin{array}{l}-1,05 * * \\
(0,479)\end{array}$ & 0,35 & $\begin{array}{l}-0,793 \\
(0,496)\end{array}$ & 0,452 & $\begin{array}{l}-0,699 \\
(0,702)\end{array}$ & 0,497 & $\begin{array}{l}-1,034 \\
(5541,8)\end{array}$ & 0,356 \\
\hline
\end{tabular}

Correspondence of the main job with the received work specialization (No, this work is not a work specialization)

Yes, this

work is fully

in line with 0,085

the

Yes, this job

is in a clos

specialized

$(0,054)$

$1,089 \quad 0,026$

$(0,057)$

1,026

$-0,118$

$(0,097)$

$0,889-0,172$

$(0,43)$

0,842

$0,079 \quad 1,082 \quad-0,019$

$(0,073)$

0,981

$-0,268 *$

$0,765 \quad-0,877$

$(0,217)$

0,416

Work is associated with nervous tension (No)

Yes, all the $-0,123 * *$

time.

Yes,

$-0,123^{* *} \quad 0,884 \quad-0,014$

$(0,072)$

0,986

$(0,138)$

0,993

$-0,011$

0,989

$0,199 *$

$(0,121)$

0,013

$(0,051)$

$(0,055)$

$(0,096)$

1,221

$1,528 * * *$

$(0,544)$

0,342

Exposure to harmful production factors in the workplace (None)

Yes, all the $-0,11$

0,11
$-0,097$

0,074

$(0,129)$

$-0,24 * *$

$-0,099 *$

$(0,085)$

0,86

$(0,109)$

1,013

$(0,535)$

sometimes.

$(0,06)$

$(0,063)$

1,077

$-0,46$

$(0,523)$

$-0,827$

$(0,515)$

Existence of other inconveniences in the workplace (No)

Yes, all the $\quad 0,153$

time.

$0,153 \quad 1,166 \quad 0,265 * * *$

Yes,

$(0,099)$

1,166

$(0,103)$

1,303

$0,359 * *$

$(0,149)$

$-0,078$

0,057

$1,046 \quad(0,064)$
in terms of the likelihood

1,064

$(0,105)$

1,432

$(0,602)$

0,279

$(0,061)$

1,058

$(0,448)$

Opinion on the safety of work in terms of the likelihood of an accident (Dangerous)

Completely $-0,445 * * *-0,766 * * *$

1

Safe enough

$0,253 * *$

$(0,199)$

$0,599 \quad-1,207$

8

$0,067-1,069-0,113$

$(0,752)$

$0,049 \quad 1,051$

Dangerous in

$0,173 *$

1,288

$(0,185)$

1,069

$-0,323$

$\begin{array}{lll}0,049 & 1,051 & -0,323 \\ (0,173) & & (0,695)\end{array}$

0,724

a way

$(0,1)$

$(0,105)$

1,073

of an accident

at work in the

$-0,672 * * *$

$0,511-0,804 * * *$

0,447

$-0,72 * *$

$0,487 \quad 1,002$

$(0,287)$

$(0,628)$

2,723

months (No)

Degree of satisfaction with wages and salaries (Not satisfied at all)

$\begin{array}{lllll}\text { Quite } & -0,311 * * * & & -0,843 * * * & 0,431\end{array}$

satisfied

$(0,143)$

0,226

$-0,665$

$0,696 \quad-0,715$
$(0,474)$

$\begin{array}{lllll}\text { Not quite } & 0,178^{*} & 1,195 & -0,004 & 0,997 \\ \text { satisfied } & (0,092) & & (0,094) & \end{array}$

$(0,126)$

Degree of satisfaction with job reliability (Not satisfied at all)
Quite $\quad 0,074$

Quite

satisfied

$(0,157)$

1,077

$-0,196$
$(0,162)$

0,822

$-0,556^{* * * *}$

0,574

$-1,838 * * *$

$(0,215)$

$0,574 \quad(0,617)$

$-0,89$

Not quite
satisfied

0,11
$(0,156)$

1,116

$-0,061$
$(0,161)$

0,941

$-0,19$

0,827

$(0,572)$

Degree of satisfaction with the duties performed (Not satisfied at all)

$\begin{array}{lllll}\text { Quite } & 0,065 & 1,067 & -0,03 & 0,971 \\ \text { satisfied } & (0,171) & 1,067) & (0,177)\end{array}$

$-0,144$

satisfied

$(0,171)$

$(0,177)$

0,971

$(0,239)$

0,866

$-0,969$

$(0,622)$

0,38

satisfied

$(0,172)$

$(0,178)$

1,178

$-0,018$
$(0,241)$

0,982

$-0,694$

$(0,648)$

0,5

Completeness of work for the entire period this year in the main and additional work (Did not work the entire period)

Worked the

entire period

(i.e., from

$-0,027$

0,973

0,03

1,03

0,024

1,025

$-0,627$

$(0,443)$

0,534

September)

Level of education (Does not have a basic general education) 


\begin{tabular}{|c|c|c|c|c|c|c|c|c|}
\hline $\begin{array}{l}\text { Personnel of } \\
\text { higher } \\
\text { qualification } \\
\text { (postgraduate) }\end{array}$ & $\begin{array}{l}0,093 \\
(1,122)\end{array}$ & 1,097 & $\begin{array}{l}-1,605 \\
(1,113)\end{array}$ & 0,201 & $\begin{array}{l}-3,67 * * \\
(1,551)\end{array}$ & 0,025 & $\begin{array}{l}-3,107 \\
(8380,8)\end{array}$ & 0,045 \\
\hline $\begin{array}{l}\text { Higher } \\
\text { (specialist, } \\
\text { master's } \\
\text { degree) }\end{array}$ & $\begin{array}{l}0,186 \\
(1,102)\end{array}$ & 1,205 & $\begin{array}{l}-1,379 \\
(1,09)\end{array}$ & 0,252 & $\begin{array}{l}-1,971 \\
(1,344)\end{array}$ & 0,139 & $\begin{array}{l}13,192 \\
(7530,2)\end{array}$ & 535931,2 \\
\hline 1 & 2 & 3 & 4 & 5 & 6 & 7 & 8 & 9 \\
\hline $\begin{array}{l}\text { Higher } \\
\text { (bachelor's } \\
\text { degree) }\end{array}$ & $\begin{array}{l}0,14 \\
(1,103)\end{array}$ & 1,15 & $\begin{array}{l}-1,468 \\
(1,092)\end{array}$ & 0,23 & $\begin{array}{l}-1,912 \\
(1,359)\end{array}$ & 0,148 & $\begin{array}{l}12,559 \\
(7530,2)\end{array}$ & 284584,2 \\
\hline $\begin{array}{l}\text { Incomplete } \\
\text { higher } \\
\text { education } \\
\text { (incomplete } \\
\text { higher } \\
\text { education), } \\
\text { completed } 3 \\
\text { years }\end{array}$ & $\begin{array}{l}-0,01 \\
(1,117)\end{array}$ & 0,99 & $\begin{array}{l}-1,285 \\
(1,108)\end{array}$ & 0,277 & $\begin{array}{l}-1,706 \\
(1,42)\end{array}$ & 0,182 & $\begin{array}{l}-3,01 \\
(8337,6)\end{array}$ & 0,049 \\
\hline $\begin{array}{l}\text { Secondary } \\
\text { vocational } \\
\text { under the } \\
\text { specialist } \\
\text { training } \\
\text { program }\end{array}$ & $\begin{array}{l}0,298 \\
(1,102)\end{array}$ & 1,347 & $\begin{array}{l}-1,08 \\
(1,089)\end{array}$ & 0,339 & $\begin{array}{l}-1,647 \\
(1,342)\end{array}$ & 0,193 & $\begin{array}{l}12,528 \\
(7530,2)\end{array}$ & 275836,3 \\
\hline $\begin{array}{l}\text { Secondary } \\
\text { vocational } \\
\text { education in } \\
\text { the skilled } \\
\text { worker } \\
\text { training } \\
\text { program }\end{array}$ & $\begin{array}{l}0,536 \\
(1,102\end{array}$ & 1,71 & $\begin{array}{l}-0,711 \\
(1,09)\end{array}$ & 0,491 & $\begin{array}{l}-0,981 \\
(1,342)\end{array}$ & 0,375 & $\begin{array}{l}13,602 \\
(7530,2)\end{array}$ & 807963,4 \\
\hline $\begin{array}{l}\text { Secondary } \\
\text { general }\end{array}$ & $\begin{array}{l}0,235 \\
(1,102)\end{array}$ & 1,265 & $\begin{array}{l}-1,112 \\
(1,089)\end{array}$ & 0,329 & $\begin{array}{l}-1,497 \\
(1,343)\end{array}$ & 0,224 & $\begin{array}{l}12,52 \\
(7530,2)\end{array}$ & 273767 \\
\hline Basic general & $\begin{array}{l}0,428 \\
(1,106)\end{array}$ & 1,534 & $\begin{array}{l}-0,829 \\
(1,094)\end{array}$ & 0,436 & $\begin{array}{l}-0,908 \\
(1,35)\end{array}$ & 0,403 & $\begin{array}{l}-1,928 \\
(7635,7)\end{array}$ & 0,145 \\
\hline \multicolumn{9}{|c|}{ Ability to lead an active life (I have no interest or desire) } \\
\hline Yes, I can. & $\begin{array}{l}-0,289 * * * \\
(0,077)\end{array}$ & 0,749 & $\begin{array}{l}-0,739 * * * \\
(0,078)\end{array}$ & 0,478 & $\begin{array}{l}-1,167 \text { *** } \\
(0,125)\end{array}$ & 0,311 & $\begin{array}{l}-1,133^{*} \\
(0,667)\end{array}$ & 0,322 \\
\hline $\begin{array}{l}\text { No, I can't, } \\
\text { my health and } \\
\text { age don't } \\
\text { allow it. } \\
\text { Limitations due }\end{array}$ & $\begin{array}{l}0,17 \\
(0,275) \\
\text { o health pro }\end{array}$ & $\begin{array}{l}1,185 \\
\text { ns withi }\end{array}$ & $\begin{array}{l}0,872 \\
(0,274) \\
\text { e last } 6 \mathrm{mo}\end{array}$ & $\begin{array}{l}2,391 \\
\text { (No, Il }\end{array}$ & $\begin{array}{l}1,627 * * * \\
(0,29) \\
\text { no limitatio }\end{array}$ & 5,088 & $\begin{array}{l}2,55 * * * \\
(0,685)\end{array}$ & 12,806 \\
\hline $\begin{array}{l}\text { Yes, } \\
\text { experienced } \\
\text { significant } \\
\text { limitations }\end{array}$ & $\begin{array}{l}-0,165 \\
(0,306)\end{array}$ & 0,848 & $\begin{array}{l}1,09 * * * \\
(0,303)\end{array}$ & 2,975 & $\begin{array}{l}3,081 * * * \\
(0,316)\end{array}$ & 21,784 & $\begin{array}{l}3,848 * * * \\
(0,6)\end{array}$ & 46,913 \\
\hline $\begin{array}{l}\text { Yes, I had } \\
\text { limitations, } \\
\text { but minor } \\
\text { ones. }\end{array}$ & $\begin{array}{l}0,736 \text { *** } \\
(0,136)\end{array}$ & 2,087 & $\begin{array}{l}1,688 * * * \\
(0,136)\end{array}$ & 5,41 & $\begin{array}{l}2,555 * * * \\
(0,157)\end{array}$ & 12,876 & $\begin{array}{l}2,143 * * * \\
(0,579)\end{array}$ & 8,523 \\
\hline $\begin{array}{l}\text { Presence of a } \\
\text { chronic } \\
\text { illness } \\
\text { established by } \\
\text { a doctor (No) }\end{array}$ & $\begin{array}{l}0,473 * * * \\
(0,154)\end{array}$ & 1,604 & $\begin{array}{l}1,717 * * * \\
(0,154)\end{array}$ & 5,57 & $\begin{array}{l}2,885 * * * \\
(0,17)\end{array}$ & 17,905 & $\begin{array}{l}2,272 * * * \\
(0,458)\end{array}$ & 9,699 \\
\hline $\begin{array}{l}\text { Was a } \\
\text { disability } \\
\text { group } \\
\text { assigned (No) }\end{array}$ & $\begin{array}{l}-0,12 \\
(0,476)\end{array}$ & 0,887 & $\begin{array}{l}0,691 \\
(0,473)\end{array}$ & 1,995 & $\begin{array}{l}1,205 * * \\
(0,483)\end{array}$ & 3,336 & $\begin{array}{l}1,46 * * * \\
(0,675)\end{array}$ & 4,306 \\
\hline $\begin{array}{l}\text { Applying for } \\
\text { outpatient } \\
\text { care this year } \\
\text { (No) }\end{array}$ & $\begin{array}{l}0,588 * * * \\
(0,066)\end{array}$ & 1,801 & $\begin{array}{l}1,032 * * * \\
(0,067)\end{array}$ & 2,807 & $\begin{array}{l}1,598 * * * \\
(0,104)\end{array}$ & 4,941 & $\begin{array}{l}1,56^{* * * *} \\
(0,518)\end{array}$ & 4,758 \\
\hline 1 & 2 & 3 & 4 & 5 & 6 & 7 & 8 & 9 \\
\hline $\begin{array}{l}\text { Calling an } \\
\text { ambulance } \\
\text { (No) }\end{array}$ & $\begin{array}{l}-0,288 * \\
(0,174)\end{array}$ & 0,75 & $\begin{array}{l}0,034 \\
(0,176)\end{array}$ & 1,035 & $\begin{array}{l}0,731 * * * \\
(0,195)\end{array}$ & 2,077 & $\begin{array}{l}0,41 \\
(0,468)\end{array}$ & 1,506 \\
\hline $\begin{array}{l}\text { Cases of } \\
\text { hospitalizatio } \\
\mathrm{n}(\mathrm{No})\end{array}$ & $\begin{array}{l}0,096 \\
(0,214)\end{array}$ & 1,101 & $\begin{array}{l}0,442 * * \\
(0,216)\end{array}$ & 1,556 & $\begin{array}{l}0,76 * * * \\
(0,233)\end{array}$ & 2,139 & $\begin{array}{l}1,288 * * * \\
(0,467)\end{array}$ & 3,626 \\
\hline
\end{tabular}




\begin{tabular}{|c|c|c|c|c|c|c|c|c|}
\hline $\begin{array}{l}\text { Needed } \\
\text { medical care, } \\
\text { but did not } \\
\text { seek it (No) }\end{array}$ & $\begin{array}{l}0,499 * * * \\
(0,059)\end{array}$ & 1,647 & $\begin{array}{l}0,791 * * * \\
(0,061)\end{array}$ & 2,206 & $\begin{array}{l}0,911 * * * \\
(0,09)\end{array}$ & 2,487 & $\begin{array}{l}1,341 \text { *** } \\
(0,392)\end{array}$ & 3,825 \\
\hline $\begin{array}{l}\text { Presence of } \\
\text { oral problems } \\
\text { (None) }\end{array}$ & $\begin{array}{l}0,112 * * \\
(0,049)\end{array}$ & 1,119 & $\begin{array}{l}0,414 * * * \\
(0,051)\end{array}$ & 1,512 & $\begin{array}{l}0,579 * * * \\
(0,083)\end{array}$ & 1,784 & $\begin{array}{l}0,34 \\
(0,364)\end{array}$ & 1,405 \\
\hline \multicolumn{9}{|c|}{ Whether the respondent smokes (I smoke more than one pack a day) } \\
\hline $\begin{array}{l}\text { Don't smoke } \\
\text { and haven't } \\
\text { smoked }\end{array}$ & $\begin{array}{l}0,634 * * * \\
(0,16)\end{array}$ & 1,885 & $\begin{array}{l}0,536^{* * * *} \\
(0,168)\end{array}$ & 1,71 & $\begin{array}{l}-0,081 \\
(0,253)\end{array}$ & 0,922 & $\begin{array}{l}-1,216 \\
(0,88)\end{array}$ & 0,296 \\
\hline $\begin{array}{l}\text { Smoked, but } \\
\text { quit }\end{array}$ & $\begin{array}{l}0,655 * * * \\
(0,169)\end{array}$ & 1,925 & $\begin{array}{l}0,494 * * * \\
(0,177)\end{array}$ & 1,639 & $\begin{array}{l}-0,04 \\
(0,266)\end{array}$ & 0,961 & $\begin{array}{l}-0,694 \\
(0,907)\end{array}$ & 0,5 \\
\hline $\begin{array}{l}\text { I smoke } \\
\text { occasionally. }\end{array}$ & $\begin{array}{l}0,677 * * * \\
(0,172)\end{array}$ & 1,968 & $\begin{array}{l}0,481 * * * \\
(0,182)\end{array}$ & 1,618 & $\begin{array}{l}0,029 \\
(0,302)\end{array}$ & 1,029 & $\begin{array}{l}-0,112 \\
(1,084)\end{array}$ & 0,894 \\
\hline $\begin{array}{l}\text { I smoke up to } \\
5 \text { cigarettes a } \\
\text { day }\end{array}$ & $\begin{array}{l}0,704 * * * \\
(0,186)\end{array}$ & 2,021 & $\begin{array}{l}0,735^{* * * *} \\
(0,195)\end{array}$ & 2,085 & $\begin{array}{l}0,189 \\
(0,317)\end{array}$ & 1,208 & $\begin{array}{l}0,244 \\
(0,244)\end{array}$ & 1,277 \\
\hline $\begin{array}{l}\text { I smoke up to } \\
10 \text { cigarettes a } \\
\text { day }\end{array}$ & $\begin{array}{l}0,749 * * * \\
(0,169)\end{array}$ & 2,116 & $\begin{array}{l}0,634 * * * \\
(0,177)\end{array}$ & 1,885 & $\begin{array}{l}0,071 \\
(0,275)\end{array}$ & 1,073 & $\begin{array}{l}-1,282 \\
(0,923)\end{array}$ & 0,277 \\
\hline $\begin{array}{l}\text { I smoke up to } \\
\text { one pack a } \\
\text { day }\end{array}$ & $\begin{array}{l}0,698 * * * \\
(0,166)\end{array}$ & 2,01 & $\begin{array}{l}0,654 * * * \\
(0,173)\end{array}$ & 1,924 & $\begin{array}{l}0,5 \\
(0,258)\end{array}$ & 1,648 & $\begin{array}{l}-0,615 \\
(0,923)\end{array}$ & 0,541 \\
\hline $\begin{array}{l}\text { Alcoholic } \\
\text { beverage use } \\
\text { (None) }\end{array}$ & $\begin{array}{l}0,351 * * * \\
(0,05)\end{array}$ & 1,42 & $\begin{array}{l}0,216^{* * * *} \\
(0,053)\end{array}$ & 1,241 & $\begin{array}{l}0,002 \\
(0,088)\end{array}$ & 1,002 & $\begin{array}{l}-0,077 \\
(0,395)\end{array}$ & 0,926 \\
\hline \multicolumn{5}{|l|}{ Chi Square } & \multicolumn{4}{|l|}{$26080^{* * *}$} \\
\hline \multicolumn{5}{|c|}{ Cox \& Snell R square } & \multicolumn{4}{|l|}{0,371} \\
\hline \multicolumn{5}{|c|}{ Nagelkerk R-square } & \multicolumn{4}{|l|}{0,438} \\
\hline \multicolumn{5}{|c|}{ McFadden's R-square } & \multicolumn{4}{|l|}{0,247} \\
\hline
\end{tabular}

\section{Results and Discussion}

Age has a significant influence on the subjective assessment of health, with each year the chances of assessing one's health lower than "very good" increasing. Men are more inclined to assess their health worse, compared to women, for all gradations of self-assessment. That is, age is a factor that increases the likelihood of a negative assessment of one's own health. Marital status has a significant impact on the self-assessment of health. Individuals who are married have a higher chance of assessing their health as good or satisfactory compared to very good. Widows or widowers are less likely to rate their health as poor, and divorced as satisfactory. That is, being in a registered marriage worsens, and being widowed improves one's self-assessment of health. The findings obtained by Wang, D. (2021), who also identified the relationship between physical and emotional neglect in the family, illness of a family member, material difficulties in the family, living in an unfavorable place negatively affects the population's assessment of health. That is, various negative factors affecting one family member negatively affect all family members. Somewhat different results were obtained by Jhang (2018), in which it was found that, over time, widowhood leads to a deterioration in a self-image of health.

The type of the main job has a significant impact only on the self-perception of health as good.
Individuals who work in the field of entrepreneurial activity, employed by individuals, and on an individual basis are less likely to do so. In other words, individuals with this type of major job have a lower risk of poor self-rated health. Working in a close occupation compared to a non-specialty job reduces the chances of judging health as poor, i.e., it reduces the likelihood of poor self-rated health.

Individuals whose jobs involve constant nervous tension are more likely to have very poor health, and less likely to have good health. In other words, this factor can be seen as a factor of lower self-rated health. Wu et al. (2019) also believe that people who report long working hours are more likely to report poor health. As part of the research, Wu et al. (2019) also found that the relationship between long working hours and self-rated health depended on the occupation. In addition, travel time and long working hours affect self-rated health. Those whose work occasionally involves exposure to harmful work factors are less likely to have a good, satisfactory, and poor assessment of their own health.

The presence of other inconveniences in the workplace is a factor in worsening selfassessment of health, increasing the chances of rating it as satisfactory and poor compared to very good for those who have inconveniences all the time. Cheng et al (2013) also revealed that 


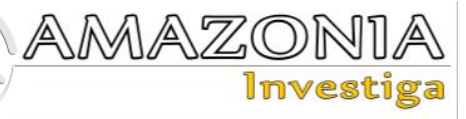

adverse factors at work have a negative impact on health assessment, and this influence only increases with age. Individuals who work in safe jobs are less likely to rate their health as good, satisfactory, or poor. That is, HAS hazardous work produced stress that worsens subjective health evaluations.

Having accidents at work reduces the chances of having a good, satisfactory, or poor assessment of one's own health.

Satisfaction with wages reduces the chances of having good, satisfactory, and poor selfassessment of health. Thus, dissatisfaction with the level of wages is a significant factor in the deterioration of an individual's assessment of his/her health.

Satisfaction with job reliability also reduces the chances of negative self-reported health (for the "poor" and "very poor" gradations). The degree of satisfaction with the duties performed, as well as the completeness of work for the entire period of the year, do not have a significant impact on self-assessment of health. Having a higher postgraduate education reduces the chances of perceiving one's health as bad. That is, a low level of education can be considered a factor of negative self-assessment of health. Similar results were obtained by Nie et al. (2021). In the study, the authors concluded that health literacy and self-assessment of one's health are correlated; the higher the literacy, the higher the self-assessment of one's health.

Having the opportunity to lead an active life reduces the chances of worsening subjective health assessment, while the lack of such an opportunity due to health and age leads to an increased chance of assessing health as bad and very bad. In other words, the lack of opportunity to lead an active life is a significant factor in the negative assessment of one's health Tebar et al (2021) also concluded that engaging in $\geq 300$ minutes of physical activity per week was associated with good self-rated health.

Having limitations due to health problems in the past six months increases the chances of a negative health rating for all grades. Being assigned a disability group increases the chances of a health rating as poor or very poor.

Individuals who have sought outpatient care in the current year are more likely to rate their health as good, satisfactory, bad, and very bad health conditions compared to very good. That is, seeking care is an indicator of poorer health.
However, with regard to calling an ambulance, the result is not so clear-cut. Calling an ambulance increases the odds of having poor health compared to very good health, and decreases the odds of having good health compared to a very good health condition.

Being hospitalized increases the odds of rating your health as satisfactory, poor, and very poor.

A significant factor is the presence of the need for medical care when an individual does not seek it for one reason or another. This fact increases the chances of deterioration of selfassessment of health.

Oral problems can lower one's own health rating from very good to good, satisfactory, and poor.

The more an individual smokes, the more likely it is that he will rate his own health worse: as good or satisfactory. Similar results were obtained by Nakata et al (2009), And secondhand smoke has the same dependence. Alcohol consumption has a similar effect as a risk factor.

\section{Conclusions}

The main factors reducing subjective health evaluations are gender, age, married status, employment, work in a profession close to the one obtained, nervous tension, harmful factors and other inconveniences in the workplace, the dangerous nature of work, dissatisfaction with wages and job reliability, inability to lead an active life, having health problems, smoking and drinking alcohol. The results obtained can be used to adjust social policy, as well as by the population in order to prevent a decline in the level of self-rated health. In addition, global research is needed to confirm the findings of this article.

\section{Bibliographic references}

Andreev, E.M., McKee, M. \& Shkolnikov, V.M. (2003). Health expectancy in the Russian Federation: a new perspective on the health divide in Europe. Bulletin of the World Health Organization, 81(11), 778 - 787. World Health Organization.

https://apps.who.int/iris/handle/10665/268835

Borim, F.S., Neri, A. L., Francisco, P. M., \& Barros, M. B. (2014). Dimensions of self-rated health in older adults. Revista de saude publica, 48(5), 714-722. https://doi.org/10.1590/s00348910.2014048005243

Borim, F., Francisco, P., \& Neri, A. L. (2017). Sociodemographic and health factors associated 
with mortality in community-dwelling elderly. Revista de saude publica, 51, 42. https://doi.org/10.1590/S1518-

8787.2017051006708

Cheng, Y., Chen, I-S., Chen, C.-J., Burr, H., \& Hasselhorn, H.M. (2013). The influence of age on the distribution of self-rated health, burnout and their associations with psychosocial work conditions. Journal of Psychosomatic Research, 74(3),

213-220. https://doi.org/10.1016/j.jpsychores.2012.12.01 7

Fafard, P., \& Cassola, A. (2020). Public health and political science: challenges and opportunities for a productive partnership. Public Health, 186 , 107-109. https://doi.org/10.1016/j.puhe.2020.07.004 Federal State Statistics Service of the Russian Federation (2019). Comprehensive monitoring of living conditions of the population in 2018 . Retrieved from: http://www.gks.ru/free_doc/new_site/KOUZ18/ index.html (in Russian).

French, D. J., Sargent-Cox, K., \& Luszcz, M. A. (2012). Correlates of subjective health across the aging lifespan: understanding self-rated health in the oldest old. Journal of aging and health, 24(8), 1449-1469.

https://doi.org/10.1177/0898264312461151

Heller, D. A., Ahern, F. M., Pringle, K. E., \& Brown, T. V. (2009). Among older adults, the responsiveness of self-rated health to changes in Charlson comorbidity was moderated by age and baseline comorbidity. Journal of clinical epidemiology, 62(2), 177-187.

Jhang, F.-H. (2018). Changes in marital status and links to self-rated health: A study of temporal relationships. The Social Science Journal, 55 (2), 87-96.

https://doi.org/10.1016/j.soscij.2017.08.001

Nakata, A., Takahashi, M., Swanson, N.G, Ikeda, T., \& Hojou, M. (2009). Active cigarette smoking, secondhand smoke exposure at work and home, and self-rated health. Public Health, 123(10), 650-656.

https://doi.org/10.1016/j.puhe.2009.09.006

Nery Guimarães, J.M., Chor, D., Werneck, G.L. et al (2012). Association between self-rated health and mortality: 10 years follow-up to the Pró-Saúdecohort study. BMC Public Health, 12, 676. https://doi.org/10.1186/1471-2458-12-676

Nie, X., Li, Y., Li, C., Wu, J., \& Li, L. (2021). The Association Between Health Literacy and Self-rated Health Among Residents of China Aged 15-69 Years. American Journal of Preventive Medicine, 60 (4), 569-578. https://doi.org/10.1016/j.amepre.2020.05.032
Nusratullin, I., Kuznetsova, S., Gazizyanova, Y., Kutsenko, E., \& Berezhnaya, L. (2020). Socioeconomic development of Russia in terms of the BRICS countries' development. Amazonia Investiga, $9(27)$, 52-61. https://doi.org/10.34069/AI/2020.27.03.6

Perlman, F., \& Bobak, M. (2008). Determinants of self rated health and mortality in Russia - are they the same?. International Journal for Equity in Health, 7(19). https://doi.org/10.1186/14759276-7-19

Puvil T., Lindenberg J., Gussekloo J., de Craen A.J.M., Slaets J.P.J., \& Westendorp R.G.J. (2016). Associations of Various Health-Ratings with Geriatric Giants, Mortality and Life Satisfaction in Older People. PLoS ONE, 11(9), article number e0163499. https://doi.org/10.1371/journal.pone.0163499 Rodrigues, D.E., César, C.C., Xavier, C.C., Caiaffa, W.T., \& Proietti, F.A. (2021). Exploring neighborhood socioeconomic disparity in selfrated health: a multiple mediation analysis. Preventive Medicine, 145, article number 106443.

https://doi.org/10.1016/j.ypmed.2021.106443

Shevchuk, O., Kucheryavenko, M., Davydenko, S., \& Babaieva, O. (2020). Implementation of the patient's right to obtain information in the concept "health and human rights". Amazonia Investiga, $\quad 9(29), \quad 288-296$. https://doi.org/10.34069/AI/2020.29.05.33 Tebar, W.R., Werneck, A.O., Silva, D.R.P., de Souza, J.M., Stubbs, B., da Silva, C.C.M., Ritti-Dias, R.M., \& Christofaro, D.G.D. (2021). Poor self-rated health is associated with sedentary behavior regardless of physical activity in adolescents - PeNSE study. Mental Health and Physical Activity, 20, article number 100384, https://doi.org/10.1016/j.mhpa.2021.100384

Verropoulou, G. (2012). Determinants of change in self-rated health among older adults in Europe: a longitudinal perspective based on SHARE data. European journal of ageing, 9(4), 305-318. https://doi.org/10.1007/s10433-012-0238-4

Wang, D. (2021). Effect of family-related adverse childhood experiences on self-rated health in childhood and adulthood_ childhood friendships as moderator. SSM - Population Health, 14, article number 100762. https://doi.org/10.1016/j.ssmph.2021.100762

Wu, W., Chen, Y., Stephens, M., \& Liu, Y. (2019). Long working hours and self-rated health: Evidence from Beijing. China. Cities, 95, article number 102401. https://doi.org/10.1016/j.cities.2019.102401 